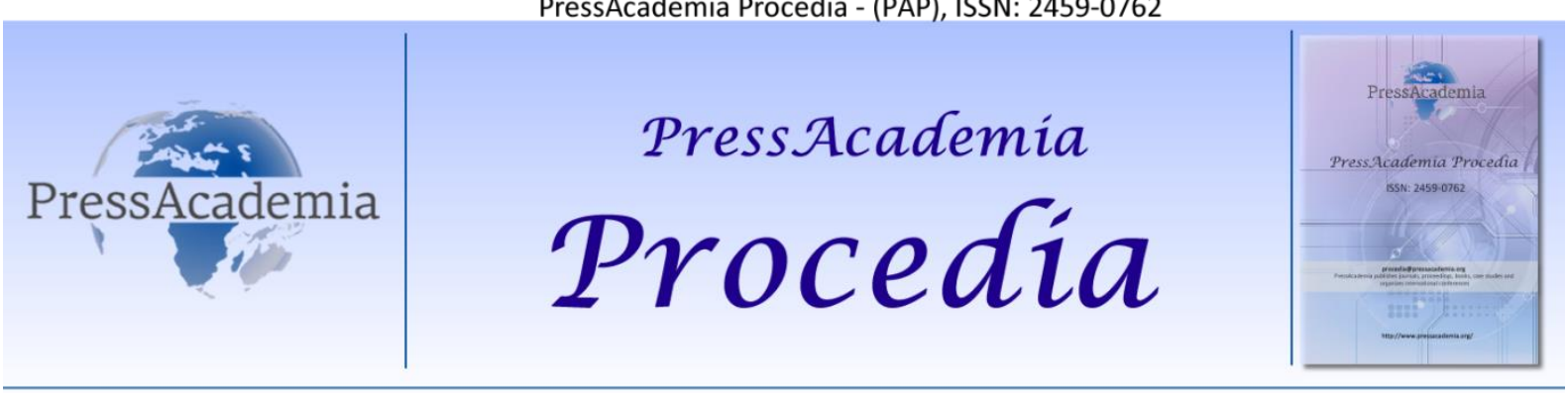

2nd World Conference on Technology, Innovation and Entrepreneurship

May 12-14, 2017, Istanbul, Turkey. Edited by Sefer Şener

\title{
A WIFI BASED RESTAURANT ORDER AUTOMATION
}

\author{
DOI: 10.17261/Pressacademia.2017.598 \\ PAP-WCTIE-V.5-2017(36)-p.262-269
}

Kerem Izzet Atam ${ }^{1}$ Tansal Gucluoglu ${ }^{2}$

${ }^{1}$ Yildiz Technical University, keremizzet.atam@stu.khas.edu.tr

${ }^{2}$ Yildiz Technical University, tansal@yildiz.edu.tr

\begin{abstract}
In traditional way of meal ordering process at a restaurant, firstly customers browse through the restaurant menu and then they wait for a waiter to come and take their orders. This procedure can be slow and decrease customer satisfaction during busy hours. Moreover, it is prone to human made mistakes. Automated systems can be used to optimize procedures by means of minimizing human made mistakes, decreasing waste of paper and thus making ordering procedures more time and cost efficient. There have been numerous attempts to automate ordering process in restaurants in recent years. For example, having a tablet PC on each restaurant table is one of the most popular solutions in the market while it is not cost effective since it requires many tablets. In this paper, we have designed and implemented an automation solution which benefits from WiFi's captive portal feature on mobile device of restaurant customers. In the proposed system, customers just need to connect restaurant's open WiFi from their table without waiting for a waiter to come and take their orders. Once their connection succeeds, customers will be directly forwarded to the web page on cloud server where they can browse through a menu and submit their orders without installing any application or any change in their mobile device. Each customer is identified by their WiFi card's MAC address which is reported to cloud server by lightweight software that runs on WiFi access point (AP) together with their received signal strength (RSSI) level and restaurant identification code. System is designed to scale easily to multiple restaurants placing configured WiFi AP on each restaurant using same cloud server. Our proposed system provides an easy to use solution for restaurant customers and a cost effective solution for restaurant owners as they can utilize their human resources more efficiently.
\end{abstract}

Keywords: Digital food ordering system, wireless food ordering system, restaurants, Wi-Fi, network

\section{INTRODUCTION}

Advancements in numerous fields of technology especially in wireless networking, cloud systems, and mobile devices have been deeply affecting our daily life routines and even changing centuries of traditions within less than quarter century. There have been developed numerous solutions in so many fields to optimize various procedures by substituting of humans tasks with automation systems. Hospitality industry is one of those areas where engineers studied to combine and utilize these technologies to develop automation mechanisms to have optimized and efficient systems in recent years.

Hence most of systems in hospitality industry are used by staffs but not customers even though customers are in the most critical part of their process. So staffs use those automation systems to work more efficiently, even though they interact with their customer in conventional way. For example think of ordering scenario described by steps in a restaurant:

\section{$\square$ Waiters visit each one of tables to have orders from customers in traditional way. \\ Then they use their hand terminal gadget to input orders to automation system. \\ $\square$ Automation system handles billing and also updates screens in kitchen.}

This is one of most common scenario because staffs are obligated and also trained to use these automation systems but customers cannot be forced to be trained to use such new systems since they are the ones who must be satisfied, especially 
in hospitality industry. In our proposed system, restaurant customer who will happen to use our system for the first time will be having ordering scenario as follows:

\section{Customer pick one of table and settles himself}

He starts to wait for the waiter to come to take his order.

$\square$ He notices paper holder with instructions simply telling him to connect WiFi point with mobile device to submit his order.

$\square$ Once he connects to WiFi AP, splash page with menu of restaurant welcomes with browser under URL of "http://menu.wf".

$\square$ He checks for the meals and adds desired ones into his basket.

$\square$ He submits his order with table code that is written on paper holder.

$\square$ Internet usage will be open to customer as he correctly enters 4 digits table code.

Customer is not required to install any application or make any change on his mobile device to use this system due to web based menu page, he also do not need to type domain name of website thanks to captive portal feature of WiFi AP which automatically directs customers to website. This system is advantageous compared to existing conventional procedures and other variants in the market by means of manpower need, likelihood of human made mistakes, capital, operational expenses and most importantly customer satisfaction. Since all it is really is for customer to submit his order via this automation and all it needs for restaurant is a WiFi AP to install system. The rest of this paper is organized as follows. Section 2 reviews the existing and new approaches that are followed in recent years. Section 3 describes system design. Section 4 shows and discusses experimental results of our implementation and finally in section $V$ we draw conclusion and future scope.

\section{LITERATURE REVIEW}

\subsection{Waiter Based Systems}

Waiter based approaches are most widely used methods where waiters have full authority to manage communication between customer and kitchen staff. It is favorable for places where social interaction between customer and restaurant staff is important. But it has so many drawbacks since it is bulky and open to human made mistakes.

\subsubsection{Pen and Paper Model}

This method and its variants are most primitive and most common procedure used in restaurants. In this system waiter uses small notepaper to write down orders. Menu cards are printed into paper. Ordering operation starts as customer sits to their table and waits for the waiter to come. Then waiter delivers menu card and waits for some time for customer to decide what to order. After waiter notes the orders he goes to kitchen part to inform kitchen staff for meals.

This traditional method has so many drawbacks in terms of efficiency and cost. System is highly depended on paper which is not suitable material for environments where foods are prepared and served. Paper can easily be affected by water, fire and oil. It is open to human made mistakes because waiters listen orders, they note them and they deliver it to kitchen. There are three people in this communication chain which makes it really likely to happen mistakes. It is common for restaurants to update price lists or making various modifications on menu cards. But even for minor edits whole menu cards need to be updated which results in paper waste and money. Waiters should check every table for customers if they are ready to order or waiting for check out. It can be even more troubling for places where there are more than few floors and architecture doesn't let staff to see every point in restaurant. It is also not easy to manage paper notes for further investigation to collect and evaluate statistics about restaurant.

\subsubsection{Kiosk Screen}

These systems are early examples hence most common systems in an attempt of digitalizing ordering process and also maintain communication between waiters and kitchen staffs. There are mostly one or few screen terminals in central positions in restaurant depends of size of place. As waiters collect orders with their notepaper, they come and input those data into screen terminals. Order information will reflected to billing and screens in the kitchen. Each screen terminals are connected to a PC running special software. 
This system makes it possible to evaluate detailed statistics about orders and understand customer tendencies for different time periods. It decreases service time as waiter doesn't need to go to kitchen to inform kitchen staff for each order. It is also less likely to have human made mistakes because it is easier to follow orders reflected into screens compared to reading from paper or keeping in mind the orders. Even though it is still bulky since waiters still need to note orders into papers and system still prone to mistakes. This system can't bring any solutions for menu cards to be easily modifiable as they are printed into papers.

\subsubsection{Wireless Mobile Terminals}

They are basically mobile versions of kiosk systems, namely personal digital assistant (PDA). They are useful as waiters do not need to note every order to paper or memorize them to re-input these information into kiosk. Waiter with PDAs can easily visit tables with it by carrying this device like a notebook. They can directly input orders and details into PDA once customers verbally inform customers.

Even though it is one of most improved technology among common automation systems for waiters in market it has some drawbacks too. Especially large restaurants with high amount of waiters require more of those devices which will lead to more expenditure. Each one of those devices continuously should function properly with enough battery level. So need for maintenance for all devices will cost time and manpower to restaurant as many pieces of hardware used in system.

\subsection{Customer Based Systems}

Customer based systems are advance version to the existing widely used automation technologies in market. In these systems customers take action first and perform operations via touch screens to submit their orders. They optimize communication chain between customer, waiter and kitchen staff. Since these systems are used by the customers they must provide easy to use and understandable interfaces because it is not practical to train each one of customer to use and teach such technologies.

\subsubsection{Mobile Application Approach}

Mobile application approach is low cost solution by means of necessary hardware as it does not require any additional device for restaurant. This method can be applied for customers to order before they come to restaurant to save time [1] or for customers inside restaurant [2]. In these systems mobile device of customers is used to submit their order. These systems expect customers to have mobile device and also expect them to install special application for restaurant. Sadly this scenario less likely to happen as long as customer is not obligated to use to order from mobile device. Because it is already a big challenge to expect customers to submit their order by themselves but in this method they also need to find this application in appstore, probably download it from internet that charged on their ISP subscription and submit order with table number which is mostly encoded into QR code. This method requires more developer work as customers might have various kinds of device beside commonly used Android and iOS based devices. There might be customers having Windows phone or even Laptop with Linux based operating system. Most of variant of such systems limit itself with Android and iOS because even struggling between versions of Operating Systems is difficult enough to maintain.

\subsubsection{Touch Screen Assembled on Table}

In this system all tables in restaurants have touch screens assembled or special casing that connects screen to table. Most of implementations use tablet computers with special application always running on it [3][4][5]. This method is favorable compared to mobile application approach as customer can easily spot those screens on table and start to use it. But installing these systems in restaurant will cost so much money. Additionally increased number of hardware will require more maintenance manpower considering all screens must work properly under condition of public use. Moreover screens under public use on food table will likely to have fingerprints of previous customer. It will either lead to customers with hygiene concerns abstain from using it or will require for waiters to clean those screen for ever service. It is likely to observe restaurants with these systems have low cost resistive touch screens which result in low quality of user experience. Because such screens have no multi touch support and screen reaction is slow.

\section{PROPOSED SYSTEM}

As it is described above waiter based ordering systems have more of workload into one person in communication chain from customer to kitchen staffs. New generation systems are trying to solve this with giving authority to customer on their order with various approach which are either not user friendly or expensive to install. In our study we designed and implemented a solution considering both user experience and cost of installing in a restaurant along with operating it with no additional effort or expenses like providing maintenance to so many pieces of hardware. Objective of our proposed 
system is to use WiFi, web and mobile technologies to address three main problem in restaurants that new generation solutions that could not able to solve :

Decrease work load on waiters in process of ordering by optimizing communication chain between customer, waiter and kitchen staff with more involvement of customers.

$\square \quad$ Provide restaurants dynamic menu card system where they can modify whenever they want and even put daily advantages instead of using printed media.

$\square \quad$ Customers no longer need to ask for Wifi password to restaurant staff as they will able to use internet once they submit their order with correct 4 digits of table code that provided to customer with Plexiglas stands on each table.

Our system is beneficial to mobile application based approach as there no need for customers to install any additional application or to do any change on their mobile device. Furthermore this system even motivates customer to use it by providing direct access to open WiFi. It is also easier to maintain our system for wide range of devices as it is developed for browsers as web site instead of specific operating system.

This solution is more cost effective solution compared to system with touch screens assembled on each tables. All it needs for our system to install in restaurant is to place WiFi AP with internet connection and provide tablet to waiter or kitchen staff to receive orders. There is no need to maintain a server computer inside restaurant as this system is cloud oriented. This will decrease maintenance work and make it easier to manage more than one restaurant from one center.

\subsection{System Design}

System has three main parts as it is depicted in Figure I; which are user devices, WiFi APs and cloud server.

\section{Figure I: System Design}

\section{Cloud Server}

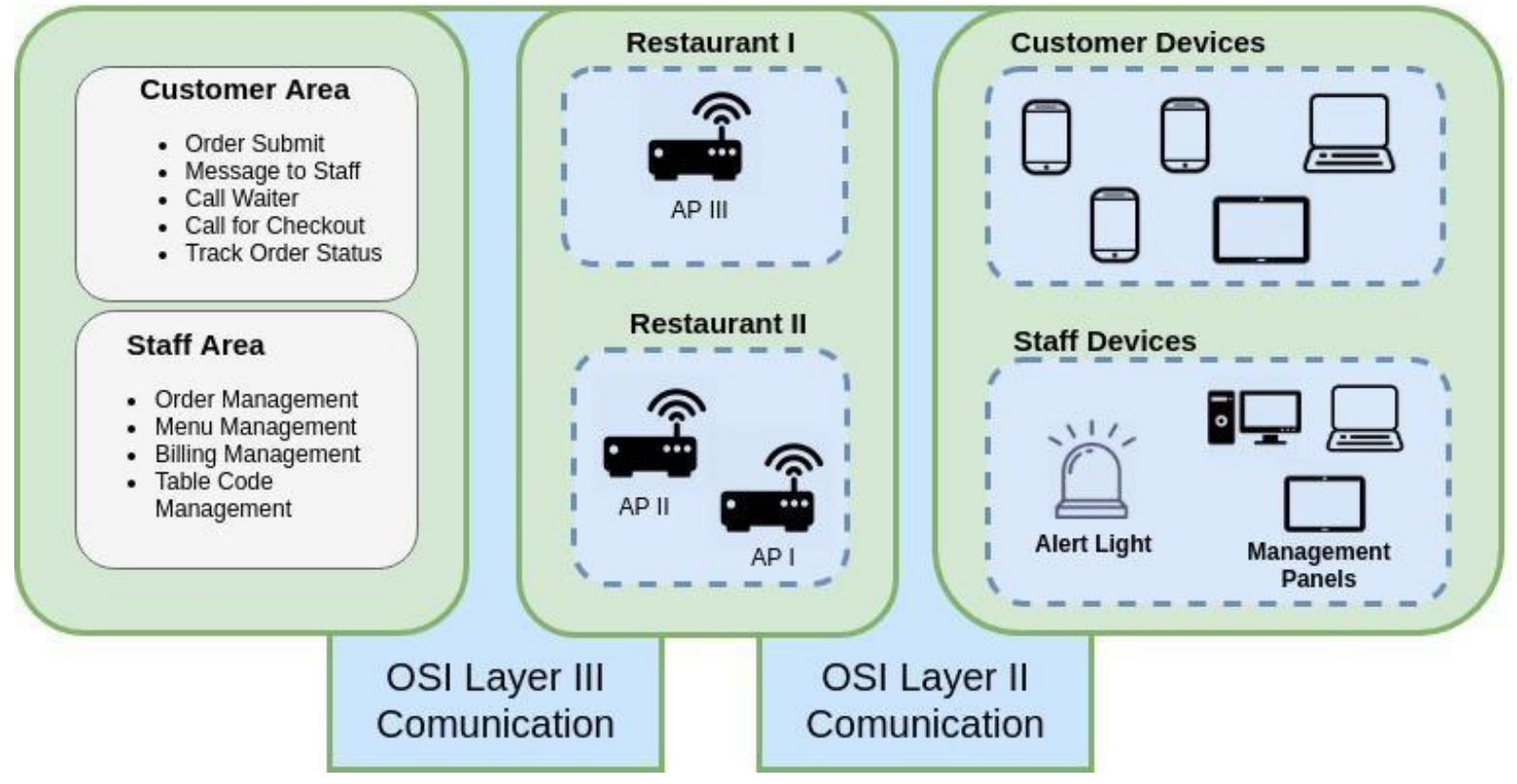

\subsubsection{Cloud System}

This is Linux based Ubuntu 14.04 PC running Apache HTTP server. This machine serves both customers and staffs for multiple restaurants. Following technologies are used in our cloud server to manage and process orders, customers, menu list and table codes for all restaurants. MongoDb is used as database software which is classified as open source document 
oriented NoSQL database program. We have prefered it as NoSQL database systems like MongoDb fits agile approach very well. Because it gives space to software to be more elastic for changes according to different business models that are used in different restaurants. PHP is used in backend of web development to process and manage all kinds of data in automation and store them into database. AngularJS and JQuery are used in frontend of web development. AngularJS was our prior choice because we consider it easier way to manipulate HTML pages compared to other Javascript frameworks. This server contains two different Uls one for restaurant customers and other one for restaurant staffs which are separated as customer and staff area respectively.

Customer Area - When customers are connected to Wifi point they will automatically be forwarded to this area of server. Then server will detect customer's restaurant by the report of AP that customer device connected. Customer UI of related restaurant will welcome afterward. Customers can browse through menu page, submit their order, track their order status, leave message to restaurant staff, call waiter and make call for checkout within this area.

Staff Area - This section will serve to restaurant staffs including managers, waiters, kitchen staff and whoever in charge of menu lists and ordering process. Only devices with pre-configured MAC addresses have access to this part of server. There is also login page to ensure security of management panel against MAC spoofing attacks. Restaurant staff can access management Uls and control menu lists to be shown to customer area and manage ordering process.

\subsubsection{WiFi APs}

Those are TP-Link devices providing wireless connection within IEEE $802.11 \mathrm{~b} / \mathrm{g} / \mathrm{n}$ protocols which operates in $2.4 \mathrm{GHz}$ carrier frequency band. Restaurant can have more than one WiFi AP to maintain full signal coverage to customers within dining area. WiFi APs carry following tasks with our software running on them:

Redirect customers to menu page of restaurant under domain name "menu.wf" once they connect to WiFi point with captive portal feature. It will let system to catch customers really quickly without need of installing any application or doing any change on mobile device.

As our system is designed to give support multiple restaurant. WiFi AP sends Restaurant ID to server so that our software that runs on cloud will know which restaurant's customer is accessing. Customers will see the menu page of related restaurant that they are in.

MAC address of WiFi card of customer's mobile device is reported to cloud server to identify each customer. MAC address is unique identifier that every WiFi card that have. Our system can identify each customer with this address without need of customer enrolling or signing in to our system.

$\square$ RSSI level of each customer device reported to server with the order submission by WiFi AP. RSSI is a digital value of measurement of the power present in a received radio signal. This information can be seen by management panel for security reason and also monitoring coverage performance of WiFi AP. This system can be used anywhere WiFi AP coverage reaches. So that some people with abusive intents might submit orders outside of restaurant. These suspicious orders can be detected by the RSSI performance as they will have weaker signal powers.

\subsubsection{User Devices}

These can be various devices from mobile phone to laptop PC that able to connect WiFi APs to access either customer area or staff area on cloud server. These devices should have WiFi card supporting IEEE $802.11 \mathrm{~b} / \mathrm{g} / \mathrm{h}$ protocols which operates in 2.4GHz carrier frequency band and able to run any kind of web browser. Today all kinds of smart device even old fashioned ones meet with such requirements.

Customer Devices - These are mobile devices that are used to access customer area on cloud server. Devices are directed to menu splash page in customer area by their connection to WiFi AP. They can view meals, submit their orders, track their order, send call to waiter with message and ask for bill to checkout within customer UI.

Staff Devices - These are equipments that are used by restaurant staff to manage order process and modify menu lists. MAC address of network interfaces that are used for this purpose are defined into cloud server for security control with the help of MAC address reporting mechanism that WiFi APs have.

Management Panels: These devices are used to access staff area of cloud server to manage ordering process and modify meal lists. These can be small tablet to desktop PC having connection to cloud server via WiFi AP. 
Alert Light: We have developed a light system in our automation in a request of restaurant staffs. As our system is alternative to their existing automation, continuously checking our system's order management panel requires extra work for them. We have designed order alert module that notifies staffs with light for the any customer call that needs action or approval by restaurant staff. We have used an open source loT platform named NodeMCU having built in ESP8266 WiFi chip with full stack TCP/IP support along with MCU.

\section{RESULTS AND DISCUSSION}

In our development we have aimed to apply agile approach which leads us to work with our customers during continuing development process to shape the system in optimum way addressing practical solutions on place. Sarayli restaurant welcomed us to install our system which is located in close vicinity of our development environment. So we could easily observe and test our system. This is big restaurant with capacity to serve 3000 people in $4000 \mathrm{~m} 2$ area. They like to test this system in garden part where their waiters having difficulty to manage that area with waiters. We have chosen 8 tables for our test setup and placed them our paper stands which contain table codes and instructions to use system. One tablet PC is placed just next to kiosk screen to receive orders as management panel.

During our tests we have surveyed with restaurant staffs and managers and ask for any kind of problem to solve and any additional feature to develop. Their first demand was full adaptation of our system to their existing automation software which was waiter based kiosk system described above in literature review section. Sadly it is not possible for us to maintain our software to work with any type of automation as we have developed this system as a generic design which should able to be applied to any restaurant. It is not scalable to develop systems that need efforts to adapt each one of restaurant having different existing automation software. Their another demand came from waiters. They have complained that they need to check management panel continuously all the time to not miss any received orders. To solve this issue we come up with wireless alert light which warns waiters for incoming orders or demands of customer. So they no longer felt to need for check this screen all the time. One more improvement request came for billing summary. Existing billing system was showing receipt per customers. But sometime it might also be asked per table total including every customers in table. We have developed detailed view in billing management section where they can view order totals per table and per customers.

We have observed our system performance by number of orders and statistics of menu page traffic during one week period. Statistics for users are obtained both within our system and by Yandex Metrica, a free service designed to measure websites visits and analysis of user behavior. In our tests; restaurant menu page viewed by 112 different devices with the bounce rate of $26.6 \%$ which means $73.4 \%$ of customers browsed through menu page and checked meals during their attempt to connect to internet via our WiFi AP. Our system received 17 of 83 orders in 8 tables during one week period which makes $20 \%$ of orders. Considering this restaurant have mainly customers from middle aged people who visit this place with their families, we name this outcome as acceptable result as it has even higher potential for places with younger customers who are more motivated to use new technology. 


\section{Figure II: Devices by Number of Sessions}

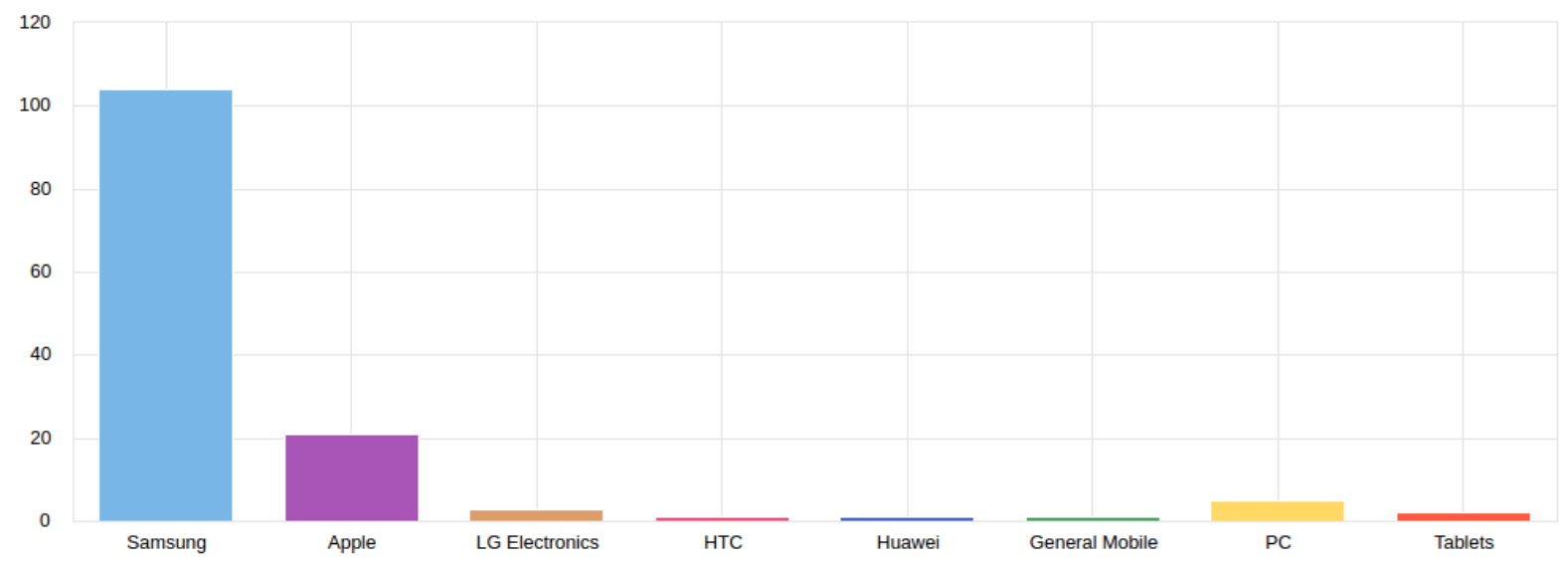

Figure III: Age Groups

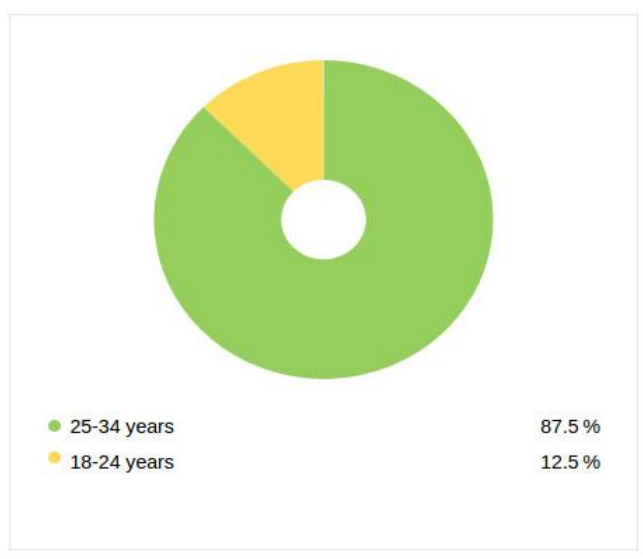

Figure IV: Device Shares

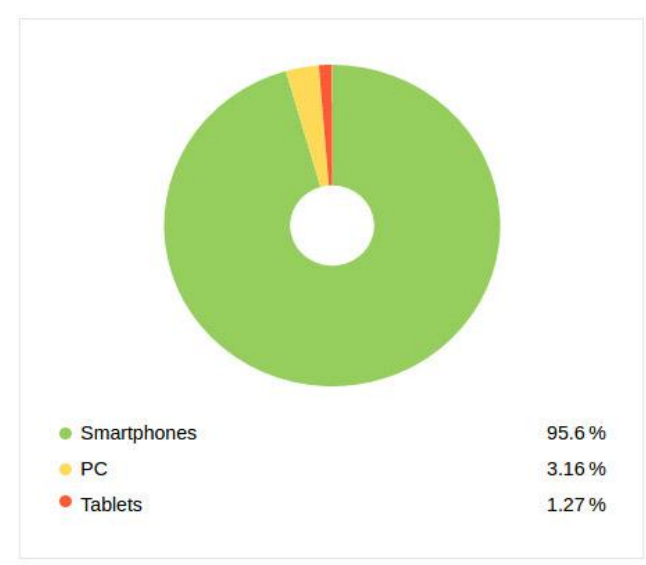

Figure III and figure IV shows share of age groups and device types respectively. Figure II depicts detail statistics of customer devices by number of sessions. Yandex Metrica reports support our claims that people who are in over aged almost never tried to use system even though majority of customers are from this age group according to our observations. Statistics also shows superiority of our system over mobile application approach explained in literature section where it is hardly possible to give support to large spectrum of devices from various vendors.

\section{CONCLUSION}

In this study, we have proposed our design and implementation of restaurant automation with different approach unlike systems in use in the market and proposed designs in literature. We have discussed advantages of our system as it attracts customers with WiFi connection and let them view menu list, submit their order, track order process, call waiter and message to restaurant staff with provided UI option on their mobile device. We aimed to have less man power need of restaurant staffs as we give more authority to customers into their ordering process.

As our project will be used by more and more in restaurants, our design is open to development for other features and ideas. This system has unique network model as this system exists in both ends of WAN communication from AP to cloud server. It brings different kinds of security threats and also makes it possible for different approaches to secure. We have used MAC based security protection but it can also be exploited by MAC spoofing attacks. To prevent this kind of abusive penetration attempts into our system, smarter methods can be used to verify staff devices using machine learning algorithms by taking usage behaviors into account to detect suspicious login attempts. 


\section{REFERENCES}

[1] Varsha Chavan, Priya Jadhav, Snehal Korade and Priyanka Teli, "Implementing Customizable Online Food Ordering System Using Web Based Application", in IJISET - International Journal of Innovative Science, Engineering \& Technology, Vol. 2 Issue 4, April 2015.

[2] Shweta Shashikant Tanpure, Priyanka R. Shidankar, Madhura M. Joshi, “Automated Food Ordering System with Real-Time Customer Feedback", in International Journal of Advanced Research in Computer Science and Software Engineering, Volume 3, Issue 2, February 2013

[3] Nibras Othman Abdul Wahid, "Improve the Performance of the Work of the Restaurant Using PC Touch Screen", in Comput Sci Syst Biol 7: 103-107. doi:10.4172/jcsb.1000144

[4] Kunal Gupta, Stuti Saxena, “DESIGN AND IMPLEMENTATION OF WIRELESS MENU CARD”, in Confluence The Next Generation Information Technology Summit (Confluence), 2014 5th International Conference

[5] Bhaskar Kumar Mishra, Tanmay Bakshi, Bhawani Singh Choudhary, "Touch Based Digital Ordering System on Android using GSM and Bluetooth for Restaurants", in India Conference (INDICON), 2015 Annual IEEE 